\title{
Características psicopatológicas y dimensiones de personalidad en pacientes adultos jóvenes dependientes del cannabis en tratamiento: un estudio comparativo
}

\section{Psychopathological symptoms and personality traits in young adult cannabis-dependent treatment seekers: A comparative study}

\author{
Ricardo Bravo de Medina ${ }^{1}$; Enrique Echeburúa; \\ JAVIER AIZPIRI ${ }^{1}$
}

\author{
(1) Medicina Psico-Orgánica, S.L. \\ (2) Facultad de Psicología \\ Enviar correspondencia a: \\ Enrique Echeburúa. Facultad de Psicología. Avda. de Tolosa, 70. \\ 20018 San Sebastián. E-mail: enrique.echeburua@ehu.es
}

\section{RESUMEN}

El objetivo de este estudio fue describir las caracteristicas psicopatológicas y dimensiones de personalidad de los pacientes adultos jóvenes dependientes del cannabis, comparándolas, a su vez, con un grupo de control clínico de pacientes psiquiátricos, pero sin diagnóstico de abuso de sustancias, y con un grupo de control sano. La muestra estuvo compuesta por 141 pacientes dependientes del cannabis en tratamiento ambulatorio, 140 pacientes no-adictos, también en tratamiento ambulatorio, y 140 sujetos sanos apareados con las muestras clínicas en sexo, edad y nivel socioeconómico. Todos los sujetos fueron evaluados con diferentes instrumentos relacionados con la personalidad (Escala de Búsqueda de Sensaciones, Escala de Impulsividad y Cuestionario de Ansiedad-Rasgo) y con diversas características psicopatológicas (SCL90-R, BDI, STAI, Escala de Inadaptación). Los pacientes pertenecientes a los grupos clínicos mostraron una mayor sintomatología ansioso-depresiva y mayores problemas de inadaptación que los sujetos del grupo de control sano, pero no había diferencias entre los grupos clínicos. Los únicos factores especificos del grupo de sujetos dependientes del cannabis fue su menor nivel formativo y la mayor presencia de ideación paranoide. Los pacientes dependientes del cannabis presentaron un mayor nivel de impulsividad y de búsqueda de sensaciones que los otros dos grupos. Por último, se comentan las implicaciones de esta investigación para la práctica clínica y para futuras investigaciones.

Palabras clave: dependencia del cannabis, dimensiones de personalidad, características psicopatológicas.
Key words: cannabis dependence, personality traits, psychopathological symptoms. 


\section{INTRODUCCIÓN}

$\mathrm{E}$ consumo abusivo de cannabis, vinculado frecuentemente a la ingesta de bebidas alcohólicas, constituye un problema de salud pública (Ministerio de Sanidad y Consumo, 2008) y puede ser causa de múltiples alteraciones físicas y psicológicas. Al margen de los síntomas psicopatológicos frecuentes en la intoxicación por cannabis (ideas delirantes paranoides, ataques de pánico, cuadros confusionales, etcétera), el consumo crónico puede generar una disminución del aprendizaje y alteraciones de la memoria a corto plazo, un deterioro en la actividad académica/laboral, pérdida de motivación por el estudio/trabajo, cambios bruscos de humor y síntomas ansioso-depresivos, así como una alteración del sistema inmunológico (Chabrol, Ducongé, Casas, Roura y Carey, 2005). Asimismo el consumo temprano del cannabis (antes de los 18 años) y a dosis altas o frecuentes puede favorecer la aparición de la esquizofrenia en personas con una predisposición genética a este trastorno (Barkus y Lewis, 2008; Núñez y Gurpegui, 2002; Zammit, Allebck, Andreasson, Lundberg y Lewis, 2002).

Al margen de que la búsqueda de ayuda terapéutica sea más tardía, este problema comienza a incubarse frecuentemente en una población adolescente (14-18 años), es decir, en una edad escolar y cuando los sujetos están en pleno desarrollo madurativo y emocional. La generalización del cannabis está asociada a la fácil disponibilidad de la droga, a la baja percepción de riesgo y a la tolerancia social a su consumo.

Si bien se ha estudiado en otras adicciones (Bravo de Medina, Echeburúa y Aizpiri, 2007), son pocos los estudios que han relacionado la vulnerabilidad psicológica del adolescente (dimensiones de personalidad) con el consumo regular de cannabis. Entre las dimensiones de personalidad estudiadas se han señalado la búsqueda de sensaciones como un factor de riesgo para el consumo (Brill, Crumpton y Grayson, 1971; Pedersen, Clausen y Lavik, 1989) o, de una forma más genérica, la inestabilidad emocional (Shedler y Block, 1990), sobre todo cuando hay una falta de habilidades para la solución de problemas sociales (Jaffee y D'Zurilla, 2009). En algunos estudios recientes llevados a cabo en nuestro medio se ha indicado que la búsqueda de sensaciones parece ser el predictor más potente de consumo de sustancias ilegales, al menos en muestras de sujetos no-clínicos (González, Saiz, Quirós y López, 2000; Martínez-Lorca y Alonso-Sanz, 2003). No se sabe si estas variables de personalidad facilitan el consumo o si constituyen específicamente un factor de riesgo para el consumo abusivo. No obstante, ha habido algunos avances recientes en este sentido, si bien se han desarrollado los estudios con sujetos-no clínicos. Así, por ejemplo, la búsqueda de sensaciones, cuando se combina con la presencia de conductas antisociales, predice tasas más altas de consumo en los consumidores de sustancias psicoactivas (Llorens, Palmer y Perelló, 2005).

El estudio de la psicopatología asociada a la dependencia al cannabis tiene una significación clínica relevante. La presencia de síntomas psicopatológicos en pacientes dependientes de cannabis está asociada a una mayor probabilidad de problemas psicosociales e interpersonales, así como a una menor adherencia al tratamiento y a una mayor dificultad en el mantenimiento de la abstinencia, tal como se ha puesto de relieve en pacientes con dependencia al alcohol (Echeburúa, Bravo de Medina y Aizpiri, 2005, 2007, 2008; Bravo de Medina, Echeburúa y Aizpiri, 2008).

El objetivo de esta investigación es doble: estudiar las dimensiones de personalidad más relevantes (búsqueda de sensaciones, impulsividad, ansiedad-rasgo) en una población adulta joven dependiente del cannabis, buscadora de ayuda terapéutica, comparada con un grupo de control clínico y con un grupo de control sano; y analizar la sintomatología psicopatológica especifica de este grupo cuando se la compara con un grupo clínico y un grupo de control. Se trata, en última instancia, de aportar datos a una parcela del conocimiento sobre la vulnerabilidad psicológica de los consumidores abusivos y sobre los efectos psicopatológicos de esta droga a largo plazo, a efectos de poder diseñar en el futuro estrategias preventivas selectivas y procedimientos terapéuticos eficaces en función de estas variables.

\section{MÉTODO}

\section{Participantes}

La muestra de esta investigación está compuesta por 421 sujetos, distribuidos en tres grupos:

1. Grupo experimental $(N=141)$. Los criterios de inclusión fueron los siguientes: a) tener más de 18 años y buscar ayuda terapéutica por problemas relacionados con trastornos derivados del consumo abusivo de cannabis; b) cumplir los criterios de dependencia del cannabis según el DSM-IV-TR (APA, 2000); y c) recibir el diagnóstico de dependencia del cannabis como diagnóstico primario.

2. Grupo de control clínico $(N=140)$. Se trata de pacientes con un trastorno mental y con un diagnóstico distinto del de dependencia de cannabis, alcohol u otras drogas. La muestra de este grupo está constituida por pacientes que acuden al mismo servicio clínico. Los diagnósticos más frecuentes están relacionados con los trastornos de ansiedad y con los trastornos del estado de ánimo.

3. Grupo de control sano $(N=140)$. Se trata de personas sanas, sin patología mental, apareadas en edad y sexo con los grupos anteriores. La muestra de esta población se ha seleccionado entre la población que no recibe tratamiento psiquiátrico ni psicológico.

Todos los pacientes incluidos en el estudio fueron atendidos en la Unidad de Salud Mental de la Clínica de Medicina Psico-orgánica (Bilbao) entre 2006 y 2009 y dieron el consentimiento informado para formar parte de este estudio. 


\section{Diseño}

Esta investigación consiste en un estudio transversal noexperimental. De acuerdo a la finalidad del estudio, se ha llevado a cabo un estudio descriptivo, con objeto de analizar el perfil psicopatológico diferencial entre los sujetos dependientes del cannabis en tratamiento y dos grupos de control (uno clínico y otro normativo).

\section{Medidas de evaluación}

\section{Entrevista general}

Al principio se realizó una entrevista individual semiestructurada para realizar el diagnóstico clínico. En esta entrevista se recogían los datos más significativos: motivo de consulta, trastorno actual, antecedentes, familia, historia personal, nivel académico y actividad laboral, relaciones sociales, hábitos de vida, consumo de otras drogas, etcétera.

\section{Dimensiones de la personalidad}

Escala de Búsqueda de Sensaciones (SSS) (Zuckerman, Eysenck y Eysenck, 1978; versión española de Pérez y Torrubia, 1986). La escala está compuesta por 40 ítems que se contestan de forma autoaplicada. La escala recoge cuatro dimensiones, que son la búsqueda de emociones (BEM), la búsqueda de excitación (BEX), la desinhibición (DES) y la susceptibilidad al aburrimiento (SAB). La fiabilidad testretest de la versión española es 0,87.

Escala de Impulsividad (BIS) (Barratt, 1985; versión española de Luengo, Carrillo de la Peña y Otero, 1991). Se trata de una escala autoaplicada que consta de 33 items y abarca tres tipos de impulsividad: impulsividad motora, impulsividad cognitiva e improvisación/ausencia de planificación. La fiabilidad de la versión española es 0,56.

Cuestionario de Ansiedad-Rasgo (STAI-R). Es una parte del cuestionario de ansiedad estado-rasgo (Spielberger, Gorsuch y Lushene, 1970; versión española de TEA, 1982). Se valora la ansiedad-rasgo como una predisposición ansiosa estable a percibir las situaciones como amenazantes. Consta de 20 ítems. El punto de corte para la población masculina es de 25 y para la población femenina de 32. La versión española de esta prueba presenta unos índices de consistencia interna entre 0,84 y 0,87 . Los valores de fiabilidad calculada por el procedimiento de las dos mitades alcanzan un valor de 0,86 . En este estudio las puntuaciones se van a expresar en centiles.

\section{Características psicopatológicas}

Listado de 90 síntomas-revisado (SCL-90-R) (Derogatis, 1983; adaptación española de González de Rivera, 2002). Es un cuestionario autoaplicado multidimensional compuesto por 90 ítems. El cuestionario proporciona información en relación a nueve dimensiones de sintomatología psicopatológica (somatización, obsesión-compulsión, sensibilidad interpersonal, depresión, ansiedad, hostilidad, ansiedad fóbica, ideación paranoide y psicoticismo), así como a tres índices generales, que son el Índice Global de Severidad (GSI), el Índice de Distrés de Síntomas Positivos (PSDI) y el Índice de Sintomatología Positiva Total (PST). Los coeficientes alfa de fiabilidad de la adaptación española del SCL-90- $R$ están entre 0,77 y 0,90. El SCL-90-R muestra una elevada sensibilidad diagnóstica, de entre el 80 y el $90 \%$, pero una muy baja especificidad, ente el 20 y el $60 \%$. En este estudio las puntuaciones se van a expresar en puntuaciones directas.

Cuestionario de Ansiedad-Estado (STAI-E). Deriva del cuestionario de ansiedad estado-rasgo (Spielberger, Gorsuch y Lushene, 1970; versión española de TEA, 1982). Se valora un estado emocional transitorio de atención tensa y aprensión, así como de hiperactividad del Sistema Nervioso Autónomo. Consta de 20 ítems. El punto de corte para la población masculina es de 28 y para la población femenina de 31. La versión española de esta prueba presenta unos indices de consistencia interna entre 0,90 y 0,93. Los valores de fiabilidad calculada por el procedimiento de las dos mitades alcanzan un valor de 0,94. En este estudio las puntuaciones se van a expresar en centiles.

Inventario de Depresión de Beck (BDI). Es un autoinforme que evalúa la presencia e intensidad de la sintomatología depresiva (Beck, Rush, Shaw y Emery, 1979; validación española de Sanz y Vázquez, 1998). La versión utilizada consta de 21 ítems. El BDI presenta una fiabilidad media alta en términos de consistencia interna (alfa total de la esca$\mathrm{la}=0,90)$. Con un punto de corte de 21 , la sensibilidad es del $75 \%$ y la especificidad del 76\% (Sanz, Perdigón y Vázquez, 2003).

Escala de Inadaptación (EI) (Echeburúa, Corral y Fernández-Montalvo, 2000). Es un instrumento de autoevaluación que consta de seis ítems, que se valoran de 0 a 5 puntos en una escala de tipo Likert. En esta escala se recogen la inadaptación global y cinco aspectos específicos referentes a la inadaptación en la vida cotidiana: trabajo/estudios, vida social, tiempo libre, relaciones de pareja y vida familiar. Las puntuaciones totales pueden oscilar entre 0 y 30, estableciéndose como puntos de corte la puntuación 12 en la escala total y la de 2 en cada uno de los ítems para discriminar los niveles clínicamente significativos de inadaptación. Esta escala tiene una buena fiabilidad (alfa de 0,94) y una adecuada validez discriminante entre pacientes y sujetos sanos $(t=25,3 ; p>$ .001) (Echeburúa et al., 2000).

\section{Procedimiento}

El diagnóstico de dependencia del cannabis ha sido llevado a cabo, con arreglo a los criterios específicos del $D S M-I V-T R$, por un psiquiatra y por un psicólogo clínico conjuntamente, ambos con una amplia experiencia clínica. El grado de concordancia entre ambos profesionales respecto al diagnóstico fue del 100\%. 
Una vez seleccionados todos los sujetos de la muestra, se les administró la batería de pruebas en 2 sesiones. La administración de las pruebas se llevó a cabo cuando todos los sujetos estaban abstinentes respecto a cualquier droga.

\section{Análisis de datos}

Para el análisis de los resultados se han utilizado pruebas paramétricas (chi-cuadrado y análisis de la varianza). Como análisis post-hoc se ha recurrido a la prueba de Bonferroni. Estos resultados se han obtenido por medio del SPSS 13.0 para Windows.

\section{RESULTADOS}

En este trabajo se han analizado los siguientes datos en los tres grupos de la muestra: a) las variables sociodemográficas; b) las características psicopatológicas; y c) las dimensiones de personalidad.

\section{Variables sociodemográficas}

Las principales características sociodemográficas de la muestra están señaladas en la tabla 1. La muestra total está constituida fundamentalmente por una mayoría de hombres jóvenes (por debajo de los 30 años) y de clase media. Como se puede observar, la única diferencia significativa entre los grupos es en el nivel educativo $\left(X^{2}=13,596 ; p<.001\right)$, con una menor frecuencia de estudios universitarios en el grupo de pacientes dependientes del cannabis.

\section{Características psicopatológicas}

Como puede apreciarse en la tabla 2, existen diferencias estadísticamente significativas en las características psicopatológicas globales del SCL-90-R entre ambos grupos de pacientes y el grupo de control sano. Sin embargo, no hay diferencias entre los dos grupos de pacientes. Sólo en la subescala de ideación paranoide se observa una diferencia significativa estadística del grupo de dependientes del cannabis con el grupo de control sano (Bonferroni $=1,02$ ) y una significación tendencial (Bonferroni $=0,27$ ) con el grupo de control clínico.

Respecto a las características psicopatológicas más específicas, la sintomatología ansioso-depresiva es mucho más alta en los grupos de pacientes que en el grupo de control sano. Tampoco en este caso hay diferencias entre los dos grupos de pacientes.

En cuanto a la adaptación a la vida cotidiana, los pacientes de ambos grupos de pacientes están peor adaptados que el grupo de control sano. Una vez más, no hay diferencias entre uno y otro grupo de pacientes.

Tabla 1. Características sociodemográficas de la muestra según los grupos

\begin{tabular}{|c|c|c|c|c|}
\hline Variables & $\begin{array}{l}\text { Grupo de dependientes del cannabis } \\
\qquad \begin{array}{c}\mathrm{N}=141 \\
\mathrm{X}(\mathrm{DT})\end{array}\end{array}$ & $\begin{array}{l}\text { Grupo de control clínico } \\
\qquad \begin{array}{c}N=140 \\
X(D T)\end{array}\end{array}$ & $\begin{array}{l}\text { Grupo de control sano } \\
\qquad \begin{array}{c}N=140 \\
X(D T)\end{array}\end{array}$ & $\mathrm{F}$ \\
\hline Edad media & $29,21(8,399)$ & $28,24(5,562)$ & $27,60(4,569)$ & 2,268 \\
\hline Variables & $\begin{array}{l}\text { Grupo de dependientes del cannabis } \\
\qquad \begin{array}{c}\mathrm{N}=141 \\
\mathrm{~N}(\%)\end{array}\end{array}$ & $\begin{array}{l}\text { Grupo de control clínico } \\
\qquad \begin{array}{c}\mathrm{N}=140 \\
\mathrm{~N}(\%)\end{array}\end{array}$ & $\begin{array}{l}\text { Grupo de control normativo } \\
\qquad \begin{array}{c}\mathrm{N}=140 \\
\mathrm{~N}(\%)\end{array}\end{array}$ & $X^{2}(g \mid)$ \\
\hline $\begin{array}{l}\text { Sexo } \\
\text { Hombre } \\
\text { Mujer }\end{array}$ & $\begin{array}{l}101(71,6 \%) \\
40(28,4 \%)\end{array}$ & $\begin{array}{l}83(59,3 \%) \\
57(40,7 \%)\end{array}$ & $\begin{array}{l}93(66,4 \%) \\
47(33,6 \%)\end{array}$ & 4,783 (2) \\
\hline $\begin{array}{l}\text { Estado civil } \\
\text { Soltero } \\
\text { Casado/conviviendo } \\
\text { Separado/divorciado }\end{array}$ & $\begin{array}{c}116(82,3 \%) \\
24(17 \%) \\
1(0,7 \%)\end{array}$ & $\begin{array}{c}114(81,4 \%) \\
23(16,4 \%) \\
3(2,1 \%)\end{array}$ & $\begin{array}{c}116(82,9 \%) \\
24(17,1 \%) \\
0(0 \%)\end{array}$ & 0,313 (2) \\
\hline $\begin{array}{l}\text { Nivel cultural } \\
\text { Estudios primarios } \\
\text { Estudios secundarios } \\
\text { Universidad }\end{array}$ & $\begin{array}{l}46(32,6 \%) \\
79(56,0 \%) \\
16(11,3 \%)\end{array}$ & $\begin{array}{l}38(27,1 \%) \\
64(45,7 \%) \\
38(27,1 \%)\end{array}$ & $\begin{array}{l}39(27,9 \%) \\
46(32,9 \%) \\
55(39,3 \%)\end{array}$ & $13,596^{* * *}(2)$ \\
\hline $\begin{array}{l}\text { Nivel socioeconómico } \\
\text { Medio-bajo } \\
\text { Medio } \\
\text { Medio-alto }\end{array}$ & $\begin{array}{l}16(11,3 \%) \\
90(63,8 \%) \\
35(24,8 \%)\end{array}$ & $\begin{array}{c}16(27,1 \%) \\
91(65 \%) \\
33(23,6 \%)\end{array}$ & $\begin{array}{c}15(10,7 \%) \\
102(72,9 \%) \\
23(16,4 \%)\end{array}$ & 1,702(2) \\
\hline
\end{tabular}

${ }^{* * *} p<.001$ 
Tabla 2 Comparación de las características psicopatológicas del grupo de los sujetos dependientes del cannabis con los grupos de control

\begin{tabular}{|c|c|c|c|c|}
\hline $\begin{array}{l}\text { CARACTERÍSTICAS } \\
\text { PSICOPATOLÓGICAS }\end{array}$ & $\begin{array}{l}\text { Grupo de dependientes del cannabis } \\
\text { X (DT)) }\end{array}$ & $\begin{array}{c}\text { Grupo de control clínico } \\
\text { X (DT) }\end{array}$ & $\begin{array}{c}\text { Grupo de control sano } \\
\text { X (DT) }\end{array}$ & $F$ \\
\hline \multicolumn{5}{|l|}{ SCL-90-R (Rango: 0-4) } \\
\hline Somatización & $1,09(0,65)$ & $1,34(0,99)$ & $0,47(0,49)$ & $35,975^{* * *}$ \\
\hline Obsesión-compulsión & $1,44(0,65)$ & $1,78(0,99)$ & $0,71(0,50)$ & $52,559^{* * *}$ \\
\hline Sens. Interpersonal & $1,31(0,84)$ & $1,53(0,98)$ & $0,55(0,43)$ & $47,607^{* * *}$ \\
\hline Depresión & $1,61(0,85)$ & $2,00(0,92)$ & $0,61(0,53)$ & $79,094^{* * *}$ \\
\hline Ansiedad & $1,36(0,71)$ & $1,76(1,02)$ & $0,44(0,46)$ & $74,790^{* * *}$ \\
\hline Hostilidad & $1,25(0,93)$ & $1,20(1,07)$ & $0,49(0,55)$ & $22,307^{* * *}$ \\
\hline Ansiedad fóbica & $0,83(0,72)$ & $1,01(0,90)$ & $0,25(0,33)$ & $31,498^{* * *}$ \\
\hline Ideación paranoide & $1,61(0,91)$ & $1,33(1,02)$ & $0,58(0,46)$ & $39,337^{* * *}$ \\
\hline Psicoticismo & $1,29(0,78)$ & $1,20(0,80)$ & $0,43(1,10)$ & $26,370^{* * *}$ \\
\hline GSI & $1,30(0,57)$ & $1,53(0,78)$ & $0,54(0,41)$ & $70,174^{* * *}$ \\
\hline PST (Rango 0-90) & $57,61(17,89)$ & $55,65(19,10)$ & $33,88(18,46)$ & $49,602^{* * *}$ \\
\hline PSDI & $2,03(0,51)$ & $2,64(2,98)$ & $1,33(0,36)$ & $13,702^{* * *}$ \\
\hline $\begin{array}{l}\text { Ansiedad }{ }^{1} \text { (STAl-E) } \\
\text { (Rango:0-100) }\end{array}$ & $59,45(25,85)$ & $64,53(31,56)$ & $38,89(21,65)$ & $25,442^{* * *}$ \\
\hline $\begin{array}{l}\text { Depresión (BDI) } \\
\text { (Rango:0-63) }\end{array}$ & $20,97(8,77)$ & $23,31(10,75)$ & $5,75(0,58)$ & $109,561^{* * *}$ \\
\hline $\begin{array}{l}\text { Inadaptación (EI) } \\
\text { (Rango:0-30) }\end{array}$ & $19,09(6,25)$ & $19,61(6,28)$ & $6,82(5,63)$ & $136,756^{* * *}$ \\
\hline
\end{tabular}

*** $p<.001$

1 En este caso las puntuaciones que figuran corresponden a los centiles

Tabla 3. Comparación de las dimensiones de personalidad del grupo de los sujetos dependientes del cannabis con los grupos de control

\begin{tabular}{|c|c|c|c|c|}
\hline $\begin{array}{l}\text { DIMENSIONES DE } \\
\text { PERSONALIDAD }\end{array}$ & $\begin{array}{l}\text { Grupo de dependientes del cannabis } \\
\text { X (DT) }\end{array}$ & $\begin{array}{l}\text { Grupo de control clínico } \\
\text { X (DT) }\end{array}$ & $\begin{array}{l}\text { Grupo de control sano } \\
\text { X (DT) }\end{array}$ & $\mathrm{F}$ \\
\hline $\begin{array}{l}\text { Ansiedad }{ }^{1} \text { (STAl-R) } \\
\text { (Rango:0-100) }\end{array}$ & $81,01(21,9)$ & $81,17(23,6)$ & $34,57(20,5)$ & $146,252^{* * *}$ \\
\hline $\begin{array}{l}\text { Impulsividad (BIS-10) } \\
\text { (Rango:0-132) }\end{array}$ & $62,72(16,8)$ & $55,91(18,7)$ & $45,26(12,3)$ & $30,016^{* * *}$ \\
\hline $\begin{array}{l}\text { Impulsividad motora } \\
\text { Impulsividad cognitiva } \\
\text { Improvisación y ausencia de planificación }\end{array}$ & $\begin{array}{l}20,40(6,8) \\
22,70(4,5) \\
20,58(6,5)\end{array}$ & $\begin{array}{l}18,24(9,1) \\
21,40(5,6) \\
16,49(7,9)\end{array}$ & $\begin{array}{l}13,23(5,6) \\
17,14(4,7) \\
15,53(4,2)\end{array}$ & $\begin{array}{l}25,560^{* * *} \\
33,925^{* * *} \\
18,154^{* * *}\end{array}$ \\
\hline $\begin{array}{l}\text { Búsqueda de sensaciones (SSS) } \\
\text { (Rango 0-40) }\end{array}$ & 20,05 (6) & $17,16(6,5)$ & $16,84(4,8)$ & $25,452^{* * *}$ \\
\hline $\begin{array}{l}\text { Búsqueda de emociones } \\
\text { Búsqueda de excitación } \\
\text { Desinhibición } \\
\text { Susceptibilidad al aburrimiento }\end{array}$ & $\begin{array}{l}6,05(3,6) \\
6,02(1,9) \\
5,27(2,2) \\
4,62(2)\end{array}$ & $\begin{array}{l}4,18(3,3) \\
5,47(1,9) \\
3,38(2,2) \\
4,27(1,9)\end{array}$ & $\begin{array}{l}4,40(1,9) \\
5,25(1,7) \\
4,14(1,4) \\
3,18(1,7)\end{array}$ & $\begin{array}{l}11,000^{* * *} \\
7,648^{* * *} \\
21,738^{* * *} \\
14,973^{* * *}\end{array}$ \\
\hline
\end{tabular}

*** $p<.001$

1 En este caso las puntuaciones que figuran corresponden a los centiles

\section{Dimensiones de personalidad}

Por lo que se refiere a las dimensiones de personalidad, los resultados aparecen descritos en la tabla 3. Los dos grupos de pacientes son más ansiosos que el grupo de control sano, pero no hay diferencias entre ellos.

Respecto a la impulsividad, los pacientes dependientes del cannabis muestran una mayor impulsividad motora y son más improvisadores y carentes de planificación que el otro grupo de pacientes (Bonferroni $=6,80$ y 4,80 , respectivamente) y que el grupo de control sano (Bonferroni=17,45 y 5,05, respectivamente). Asimismo el grupo de pacientes dependientes del cannabis presenta una mayor búsqueda de sensaciones, una mayor búsqueda de emociones, una mayor búsqueda de excitación y una mayor desinhibición que el otro grupo de pacientes (Bonferroni=4,88, 1,86, 0,75 y 1,88, respectivamente) y que el grupo de control sano (Bonferro$\mathrm{ni}=5,20,1,65,0,97$ y 1,13 , respectivamente). 


\section{DISCUSIÓN}

Aun siendo abundantes los estudios sobre los efectos del cannabis en los consumidores abusivos, es escasa la investigación que enfoca este problema desde la perspectiva de la vulnerabilidad psicológica de los sujetos dependientes (dimensiones de personalidad) y de los efectos a largo plazo en consumidores abusivos buscadores de ayuda terapéutica. De este modo, este estudio ha tratado de precisar las dimensiones de personalidad más específicas de los sujetos dependientes de esta droga, así como la sintomatología psicopatológica asociada al consumo crónico.

El análisis de resultados sugiere que los consumidores dependientes del cannabis tienen un menor nivel formativo que los sujetos de los otros grupos, lo que confirma la relación negativa existente entre el consumo crónico de cannabis y el rendimiento académico, bien porque el bajo rendimiento académico precede al consumo de cannabis (Hall y Solowij, 1998) o porque éste interfiere negativamente en el funcionamiento escolar por medio del síndrome amotivacional, del daño cognitivo generado o del entorno social frecuentado (Lynskey y Hall, 2000).

Desde la perspectiva de la vulnerabilidad psicológica, los sujetos dependientes del cannabis no presentan una ansiedad-rasgo específica, pero, sin embargo, son más impulsivos y más buscadores de sensaciones que otro tipo de pacientes y que los sujetos sanos. Ello concuerda parcialmente con la bibliografía previa, especialmente por lo que se refiere a la búsqueda de sensaciones (Brill et al., 1971; Pedersen et al., 1989). Estas caracteristicas de personalidad aparecen también en otro tipo de adictos (Bravo de Medina et al., 2007, 2008; Fernández-Montalvo y Echeburúa, 2004, 2006). La aportación de este estudio es que, a diferencia de otros estudios con sujetos no-clínicos (González et al., 2000; Llorens et al., 2005; Martínez-Lorca y Alonso-Sanz, 2003), los pacientes estudiados han sido sujetos dependientes del cannabis en tratamiento.

A un nivel psicopatológico, el grupo de sujetos consumidores de cannabis presenta mayor psicopatología ansioso-depresiva y mayor inadaptación a la vida cotidiana que el grupo de sujetos sanos, pero no se diferencia en estas variables del otro grupo clínico (Chabrol et al., 2005). El único síntoma especifico que diferencia a los dependientes del cannabis del resto de los pacientes es la ideación paranoide, lo que confirma parcialmente las conclusiones obtenidas en algunos estudios previos (Barkus y Lewis, 2008; Goulding, McLure-Tone y Compton, 2009).

Este estudio, aun teniendo un carácter exploratorio, aporta un enfoque novedoso que requiere continuidad en investigaciones futuras, especialmente por lo que se refiere al diseño de estrategias preventivas especificas orientadas a la población adolescente de mayor vulnerabilidad psicológica y a la implementación de programas de intervención orientados especificamente a los sintomas caracteristicos de los sujetos dependientes del cannabis. Sin embargo, algunas limitaciones de este estudio son que no se ha estudiado específicamente el policonsumo (sólo la dependencia del cannabis), que se trata de una muestra no aleatoria y que el tamaño de la muestra, aun siendo considerable, no es lo suficientemente amplio como para obtener conclusiones epidemiológicas consistentes. El estudio presentado es transversal, no longitudinal, por lo que no es posible determinar si los rasgos, como la impulsividad, 0 los sintomas evaluados son causa o consecuencia de la adicción (Perry y Carroll, 2008; Wit, 2009). Asimismo en este trabajo se ha recurrido a una muestra de pacientes dependientes del cannabis en tratamiento, lo que limita el alcance de las conclusiones. A un nivel conceptual, convendría ampliar el concepto de vulnerabilidad psicológica, limitado en este estudio a las variables de personalidad, a una perspectiva más interactiva (por ejemplo, en relación con el modelo de diátesis-estrés) y analizarlo en función de los factores de riesgo y de protección. Otros estudios futuros deberian abordar estas limitaciones.

\section{REFERENCIAS}

Barkus, E. y Lewis, S. (2008). Schizotypy and psychosis-like experiences from recreational cannabis in a non-clinical sample. Psychological Medicine, 38, 1267-1276.

Barratt, E.S. (1985) Impulsiveness defined within a systems model of personality. In E. P. Spielberger y J. N. Butcher (Eds.). Advances in Personality Assessment (pp. 113-132). Hillsdale: Lawrence Erlbaum Associates.

Beck, A.T., Rush, A.J., Shaw, B.F. y Emery, G. (1979). Cognitive therapy of depression. New York: Guilford Press.

Bravo de Medina, R., Echeburúa, E. y Aizpiri, J. (2008). Diferencias de sexo en la dependencia del alcohol: dimensiones de personalidad, características psicopatológicas y trastornos de personalidad. Psicothema, 20, 218-223.

Bravo de Medina, R., Echeburúa, E. y Aizpiri, J. (2007). Características psicopatológicas y dimensiones de personalidad en los pacientes dependientes del alcohol: un estudio comparativo. Adicciones, 19, 373-382.

Brill, N.W., Crumpton, E. y Grayson, H.M. (1971). Personality factors in marijuana use: a preliminary report. Archives of General Psychiatry, 191, 1631-165.

Chabrol, H., Ducongé, E., Casas, C., Roura, C. y Carey, K.B. (2005). Relations between cannabis use and dependence, motives for cannabis use and anxious, depressive and borderline symptomatology. Addictive Behaviors, 30, 829-840.

Derogatis, L.R. (1983). SCL-90-R. Adiministration, scoring and procedures manual. Baltimore: Clinical Psychometric Research.

Derogatis, L.R. (2002). SCL-90-R. Cuestionario de 90 sintomas. Manual. Madrid: Tea Ediciones.

Echeburúa, J., Bravo de Medina, R. y Aizpiri, J. (2005). Alcoholism and personality disorders: An exploratory study. Alcohol and Alcoholism, 40, 323-326.

Echeburúa, J., Bravo de Medina, R. y Aizpiri, J. (2007). Comorbidity of alcohol dependence and personality disorders: A comparative study. Alcohol and Alcoholism, 42, 618-622.

Echeburúa, J., Bravo de Medina, R. y Aizpiri, J. (2008). Variables de personalidad, alteraciones psicopatológicas y trastornos de personalidad en pacientes con dependencia de alcohol en función de la tipología de Cloninger. Psicothema, 20, 525-530. 
Echeburúa, J., Bravo de Medina, R. y Aizpiri, J. (2009). Personality disorders among alcohol-dependent patients manifesting or not manifesting cocaine abuse: A comparative pilot study. Substance Use \&t Misuse, 44, 981-989.

Echeburúa, E., Corral, P. y Fernández-Montalvo, J. (2000). Escala de inadaptación (El): Propiedades psicométricas en contextos clínicos. Análisis y Modificación de Conducta, 26, 325-340.

Fernández-Montalvo, J. y Echeburúa, E. (2004). Pathological gambling and personality disorders: An exploratory study with the IPDE. Journal of Personality Disorders, 18, 500-505.

Fernández-Montalvo, J y Echeburúa, E. (2006). Juego patológico y trastornos de personalidad: un estudio piloto con el MCMI-II. Psicothema, 18, 453-458.

González, M.P., Sáiz, P.A., Quirós, M. y López, J.L. (2000). Personalidad y uso-abuso de cannabis. Adicciones, 12, (Sup/ 2), 185-196.

Goulding, S.M., McClure-Tone, E.B. y Compton, M.T. (2009). Schizotypy and nicotine, alcohol, and cannabis use in a non-psychiatric simple. Addictive Behaviors, 34, 374-379.

Hall, W. y Solowij, N. (1998). Adverse effects of cannabis. The Lancet, 352, 1611-1616.

Jaffee, W.B y D'Zurilla, T.J. (2009). Personality, problem solving, and adolescent substance use. Behavior Therapy, 40, 93-101.

LLorens, N., Palmer, A. y Perelló, M.J. (2005). Caracteristicas de personalidad en adolescentes como predictores de la conducta de consumo de sustancias psicoactivas. Trastornos adictivos, 7, 90-96.

Luengo, M.A., Carrillo de la Peña, M.T. y Otero, J.M. (1991). The components of impulsiveness: A comparison of the Impulsiveness Questionnaire and the Barratt Impulsiveness Scale. Personality and Individual Differences, 12, 657-667.

Lynskey, M. y Hall, W. (2000). The effects of adolescent cannabis use on educational attainment: a review. Addiction, 95, 1621-1630.

Martinez-Lorca, M. y Alonso-Sanz, C. (2003). Búsqueda de sensaciones, autoconcepto, asertividad y consumo de drogas. ¿Existe relación? Adicciones, 15, 145-158.

Ministerio de Sanidad y Consumo (2008). Encuesta Domiciliaria sobre el consumo de Alcohol y Drogas (EDADES) 2007-2008.
Recuperado el 20 de marzo de 2009 de http://www.pnsd.msc.es/ Categoria2/observa/pdf/InformeEdades2007-2008.pdf

Núñez, L.A. y Gurpegui, M. (2002). Cannabis-induced psychosis: a cross-sectional comparison with acute schizophrenia. Acta Psychiatrica Scandinavica, 105, 173-178.

Pedersen, W., Clausen, S.E. y Lavik, N.J. (1989). Patterns of drug use and sensation-seeking among adolescents in Norway. Acta Psychiatrica Scandinavica, 79, 386-390.

Pérez, J. y Torrubia, R. (1986). Fiabilidad y validez de la versión española de la escala de búsqueda de sensaciones (forma $\mathrm{V}$ ). Revista Latinoamericana de Psicología, 18, 7-22.

Perry, J.L. y Carroll, M.E. (2008). The role of impulsive behavior in drug abuse. Psychopharmacology, 200, 1-26.

Sanz, J. Perdigón, A.L. y Vázquez, C. (2003). Adaptación española del Inventario para la Depresión de Beck-II (BDI-II): Propiedades psicométricas en población general. Clínica y Salud, 3, 249-280.

Sanz, J. y Vázquez, C. (1998). Fiabilidad, validez y datos normativos del inventario para la depresión de Beck. Psicothema, 2, 303-318.

Shedler, J. y Block, J. (1990). Adolescent drug use and psychological health: a longitudinal inquiry. American Journal of Psychiatry, 125, 370-378.

Spielberger, C.D., Gorsuch, R.L. y Lushene, R.E. (1970). STAI. Manual for the State-Trait Anxiety Inventory (Self-Evaluation Questionnaire). Palo Alto, California: Consulting Psychologists Press (versión española de TEA, 3a edic., 1982).

Wit, H. (2009). Impulsivity as a determinant and consequence of drug abuse: A review of underlying processes. Addiction Biology, 14, 22-31.

Zammit, S., Allebeck, P., Andreasson, S., Lundberg, I. y Lewis, G. (2002). Self reported cannabis use as a risk factor for schizophrenia in Swedish conscripts of 1969: historical cohort study. British Medical Journal, 325, 1183-1184.

Zuckerman, M., Eysenck, S.B., y Eysenck, A.J. (1978). Sensation Seeking in England and American: cross-cultural age and sex comparisons. Journal of Consulting and Clinical Psychology, 46, 139-149. 
